\title{
Funções gerenciais do enfermeiro no cotidiano em serviço de hemoterapia de um
}

\section{hospital universitário}

\author{
Nurse's managerial functions in the daily life of a hemotherapy service at a university hospital \\ Funciones directivas del enfermero en la vida diaria de un servicio de hemoterapia en un hospital \\ universitario
}

\section{Resumo}

Objetivos: Conhecer como o Enfermeiro utiliza as funções de gerência no cotidiano em Serviço de Hemoterapia de um hospital Universitário. Método: Estudo descritivo, com uma abordagem qualitativa, tendo como cenário um Serviço de Hemoterapia de um Hospital Universitário da rede pública estadual. A coleta de dados foi realizada por meio de uma entrevista semiestruturada e optou-se por tratar os dados pela análise de conteúdo de Bardin. Resultados: Após a transcrição e análise das entrevistas foi possível identificar quais são as funções gerenciais que enfermeiros diariamente executam para que as suas equipes possam realizar o atendimento aos pacientes em sua plenitude. Considerações Finais: os enfermeiros, onde ocorreu o presente estudo, atuam de forma a prover todo o apoio necessário para a correta consecução das atividades, atuando: na elaboração das escalas; na realização de treinamentos; na gestão dos insumos; na gestão dos possíveis conflitos; e na adoção de ações voltadas a motivar as equipes.

Palavras-chave: Enfermagem; Serviço de hemoterapia; Gerência; Ciclo do sangue.

\begin{abstract}
Objectives: To know how the Nurse uses management functions in daily life in the Hemotherapy Service in a university hospital. Method: Descriptive study, with a qualitative approach, with the setting of a Hemotherapy Service of a University Hospital of the state public network. Data collection was carried out through a semi-structured interview and it was decided to treat the data using Bardin's content analysis. Results: After transcribing and analyzing the interviews, it was possible to identify the managerial functions that nurses perform on a daily basis so that their teams can fully care for patients. Final Considerations: the nurses, where this study took place, act in order to provide all the necessary support for the correct performance of the activities, acting: in the elaboration of the scales; in conducting training; in the management of inputs; in the management of possible conflicts; and in the adoption of actions aimed at motivating teams.
\end{abstract}

Keywords: Nursing; Hemotherapy service; Management; Blood cycle. 


\begin{abstract}
Resumen
Objetivos: Conocer cómo la Enfermera utiliza las funciones de gestión en la vida diaria en el Servicio de Hemoterapia. Método: Estudio descriptivo, con abordaje cualitativo, en el marco de un Servicio de Hemoterapia de un Hospital Universitario de la red pública estatal. La recogida de datos se realizó mediante entrevista semiestructurada y se decidió tratar los datos mediante el análisis de contenido de Bardin. Resultados: Luego de la transcripción y análisis de las entrevistas, fue posible identificar las funciones gerenciales que las enfermeras desempeñan en el día a día para que sus equipos puedan atender integralmente a los pacientes. Consideraciones finales: las enfermeras, donde se realizó este estudio, actúan con el fin de brindar todo el apoyo necesario para el correcto desempeño de las actividades, actuando: en la elaboración de las escalas; en la realización de formación; en el manejo de insumos; en el manejo de posibles conflictos; y en la adopción de acciones encaminadas a motivar a los equipos.
\end{abstract}

Palabras clave: Enfermería; Servicio de hemoterapia; Manejo; Ciclo sanguíneo.

\title{
1. Introdução
}

Nas últimas décadas vem crescendo o uso de sangue e hemocomponentes como elementos básicos aplicados no tratamento de diversas doenças que afetam a população, fazendo com que a transfusão de sangue tenha se tornado uma terapia amplamente utilizada em todo o mundo, elevando a demanda por produtos hemoterápicos (Rohr et al., 2012).

A hemoterapia é um segmento que tem se destacado como uma relevante área de estudo porque engloba um expressivo emprego de recursos materiais e humanos. Entretanto, como qualquer ramo da saúde necessita investir em procedimentos que visem à identificação das falhas, buscando um incremento na qualidade dos serviços de saúde prestados a população, tendo como meta a conformidade com as normas e regulamentos vigentes (Almeida et al., 2011).

A terapia transfusional é um procedimento de natureza complexa que necessita da atuação de profissionais especializados e de notório conhecimento não apenas dos assuntos afetos a qualidade e segurança do sangue, mas também na identificação das complicações que podem ocorrer durante o Ato Transfusional, sendo as reações adversas mais comuns às alérgicas e febris e as incomuns como a lesão pulmonar aguda associada à transfusão (Barbosa et al., 2011; Faquetti et al., 2014; Buozi et al., 2019).

O Serviço de Hemoterapia é um local destinado a: prestar assistência hemoterápica e/ou hematológica; atuar na captação de doadores; realizar o processamento do sangue; implementar todos os testes sorológicos visando a segurança do processo de transfusão do sangue e hemocomponentes, buscando à prevenção de erros; armazenar e preparar as transfusões dos hemocomponentes; orientar os pacientes quanto ao processo transfusional; proceder com o ato transfusional, tendo atenção aos parâmetros a serem seguidos para a correta realização do procedimento; atuar no atendimento às reações transfusionais adversas, documentando todo o processo; e realizar a Hemovigilância (Santos et al., 2013; Beserra et al., 2014; Carneiro et al., 2017; Torres et al., 2021).

Para que todas as atividades afetas aos cuidados com o paciente ocorram de forma correta, o enfermeiro nos Serviços de Hemoterapia necessita desenvolver funções gerenciais fundamentais para que a assistência ocorra com qualidade e segurança para o paciente, por intermédio da coordenação e avaliação do trabalho voltado à gestão das atividades realizadas no referido Serviços, tais como: na elaboração de escala; na supervisão das atividades desenvolvidas pelos profissionais de enfermagem; na emissão e elaboração de relatórios; e participando dos processos de aquisição de materiais e equipamentos a serem utilizados na Hemoterapia. Cabe destacar que todas as atividades gerenciais em Serviços de Hemoterapia devem ser executadas por profissionais de enfermagem especializados, com conhecimentos nos processos relacionados com o ciclo do sangue (Frantz et al., 2021).

Nos mais variados serviços de saúde, a atuação do Enfermeiro de forma gerencial tem se apresentado primordial para que o mesmo contribua na articulação entre os vários profissionais da equipe e na organização do processo de trabalho, uma vez que o desempenho do Enfermeiro de forma gerencial, na atualidade, está em contínua transformação devido aos constantes estudos e teorias que embasam cientificamente o gerenciamento na enfermagem, pois estes profissionais devem ser capazes de 
gerenciar não apenas a assistência, mas também os recursos humanos e materiais empregados na mesma, buscando atingir um conjunto de conhecimentos, habilidades, práticas e atitudes que sejam capazes de garantir qualidade na assistência ao paciente (Grecco, 2004; Spagnol, 2005; Sanches, Christovam et al., 2006; Ferracioli et al., 2020).

No que tange a recursos materiais, os Enfermeiros necessitam atuar gerenciando a previsão, provisão, manutenção e controle dos materiais, insumos e equipamentos empregados na assistência direta e indireta ao paciente (Grecco, 2004).

Com isso. Vê-se que o Enfermeiro ocupa um lugar de destaque entre os profissionais da saúde, pois atua em diversas áreas, executando atividades de coordenação, planejamento de processos e atividades assistenciais de forma sinérgica e concatenada (Frantz et al., 2020).

Neste contexto, o objeto deste estudo é conhecer como o Enfermeiro utiliza as funções de gerência no cotidiano em Serviço de Hemoterapia de um hospital Universitário estadual, localizado no município do Rio de Janeiro/RJ.

\section{Metodologia}

O estudo é do tipo descritivo e utilizou uma abordagem qualitativa, tendo sido realizado em um Serviço de Hemoterapia, classificado como Núcleo de Hemoterapia, vinculado a um hospital universitário da rede pública estadual, localizado no município do Rio de Janeiro-RJ.

O Serviço de Hemoterapia estudado é composto por seis enfermeiros e todos aceitaram participar da pesquisa assinando o Termo de Consentimento de forma Livre e Esclarecido. Cabe ressaltar que todos os participantes exercem funções gerenciais no referido Serviço.

As entrevistas foram agendadas de forma prévia, por telefone, levando em consideração a disponibilidade, visando uma maior comodidade para os participantes da pesquisa, tendo sido adotados todos os protocolos sanitários, orientados pelo Ministério da Saúde, com relação a pandemia da COVID-19.

Os dados oriundos da pesquisa foram coletados por intermédio de uma entrevista semiestruturada, composta por questões afetas às funções gerenciais que um enfermeiro pode vir a desenvolver em um Serviço de Hemoterapia, sendo a análise dos dados obtidos realizada por meio da técnica de análise de conteúdo de Bardin (2016), e com os participantes sendo identificados com a letra E seguido de um número, de acordo com a ordem das entrevistas, para garantir o anonimato dos mesmos.

O estudo foi submetido à Plataforma Brasil e autorizado pelo Comitê de Ética da Universidade Federal do Estado do Rio de Janeiro, CAAE 31986920.0.0000.5285 e Parecer CEP nº 4.110.190, e foram seguidas as normas e recomendações relativas à pesquisa envolvendo seres humanos, contida na Resolução 446/12 do Conselho Nacional de Saúde, bem como foi recebida à anuência do Serviço de Hemoterapia, cenário do estudo, para a realização do mesmo.

\section{Resultados e Discussão}

Após a leitura criteriosa e a análise do material transcrito, observou-se a frequência de ocorrência das evocações espontâneas, ordenadas por frequência simples de ocorrência, que foram agrupadas dando origem a categoria: Funções Gerenciais do Enfermeiro no Cotidiano de um Serviço de Hemoterapia em um Hospital Universitário.

As funções gerenciais desenvolvidas pelos enfermeiros em seus ambientes de trabalho buscam fornecer aos membros de suas equipes meios para que os mesmos possam dar a continuidade na prestação dos cuidados com os doadores e pacientes.

Em um estudo realizado por Frantz et al. (2021), referente as atividades desempenhadas pelos enfermeiros em Serviços de Hemoterapia, pode-se constatar que estes profissionais desenvolvem atividades gerenciais relacionadas com as funções administrativas e da gestão das equipes e dos insumos a serem empregados nas mais diversas atividades 
desempenhadas pelo Serviço.

Conforme destacado por Frantz et al. (2021), o enfermeiro que atua gerenciando os Serviços de Hemoterapia exerce suas funções preocupando-se com todos os fatores que possam interferir na prestação do cuidado desde o doador, até o paciente que receberá o hemocomponente.

$\mathrm{Na}$ fala da enfermeira (E04) entrevistada, é possível observar o destaque dado ao gerenciamento dos materiais aplicados nos Serviços de Hemoterapia, que são específicos e demandam conhecimento peculiar.

[...] Outro ponto de destaque é o gerenciamento dos materiais em comodato, das manutenções preventivas das necessidades de reparo imediato, tem toda parte gerencial de material. (E04)

O gerenciamento de recursos materiais envolve a busca pela garantia de que os mais variados insumos empregados de forma direta ou indireta nos cuidados dos pacientes estejam sempre disponíveis para pronto uso pelas equipes de enfermagem (Ventura, Freire, \& Alves, 2016).

Os equipamentos e insumos empregados em Serviços de Hemoterapia são dotados de especificidades inerentes às atividades desempenhadas nestes Serviços, devendo o Enfermeiro atuar diretamente na previsão, provisão e manutenção destes itens que são primordiais para o correto funcionamento destes Serviços, visando à segurança dos doadores e na produção dos hemocomponentes que serão oportunamente usados no atendimento as demandas dos pacientes.

$\mathrm{Na}$ equipe de enfermagem, a responsabilidade em assumir as atividades gerenciais compete ao enfermeiro, cabendo a estes profissionais as ações de coordenação da equipe de técnicos e auxiliares de enfermagem, bem como a condução e viabilização do processo cuidativo, tendo como princípio que fundamentam as suas ações o direito da população à saúde integral, de forma digna, segura e ética (Spagnol, 2005).

Em conformidade com o estudo, observa-se que o cenário gerencial do enfermeiro nos Serviços de Hemoterapia não é diferente das outras áreas de atuação da enfermagem, sendo notório o envolvimento com equipe, escalas, insumos, conforme observado no discurso da enfermeira (E06) a seguir:

[...] Começa pelo gerenciamento da equipe de enfermagem, produção de escalas, gerenciamento de conflitos, sempre precisa e agora estou bem envolvida na questão dos insumos. (E06)

Segundo Ferracioli et al. (2020), os enfermeiros que atuam no gerenciamento de serviços hospitalares exercem suas atividades buscando a análise de situações e informações que permitirão a adoção das mais eficazes decisões. Em sua formação, o enfermeiro é capacitado para ser assertivo em suas decisões o que o auxilia na diminuição das chances de escolher soluções tendenciosas e ineficazes. Logo, o uso de ferramentas gerenciais pode permitir a análise do problema de forma sistematizada, reduzindo a margem de erro e tornando o profissional apto ao enfrentamento de novas situações.

A argumentação do autor é evidenciada no discurso da enfermeira (E05), onde se pode notar o desenvolvimento de suas atividades gerenciais no âmbito do Serviço de Hemoterapia.

[...] Estamos sempre observando e analisando a forma certa de trabalhar, avaliando e organizando, livre para tomada de decisão. (E05)

Para Lopes, Pontelli e Oliveira (2018), as principais dificuldades enfrentadas pelos enfermeiros que exercem funções de gerência estão associadas com as seguintes temáticas: convivência entre indivíduos de diferentes personalidades; falta de comunicação; companheirismo; trabalho em equipe, escassez de recursos e materiais; sobrecarga de atividade; deficit na formação profissional focada nos cuidados assistenciais; e falta de capacitação para ações gerenciais. $\mathrm{O}$ que traz argumentos 
que corroboram no destaque da importante participação do enfermeiro gerente, na mediação dos conflitos.

Neste contexto, pode-se destacar que os elementos da função gerencial que buscam a manutenção da harmonia e do equilíbrio estão presentes no discurso da enfermeira participante do estudo (E02).

[...] Acho que o diálogo é sempre a melhor maneira. Diálogo sempre. Aconteceu alguma coisa que desagradou, ou não foi bom para o serviço, sentar e dialogar. (E02)

Considerando a importância do Enfermeiro gerente, atuando em situações de conflito, observa-se que esses profissionais devem ser capazes de identificar, analisar e conduzir os atritos no seu ambiente de trabalho, de forma que os mesmos não interfiram nas atividades assistências desempenhadas.

A comunicação é uma ferramenta importante nas relações de trabalho entre a equipe multiprofissional e entre profissionais de enfermagem na assistência ao doador de sangue e o receptor dos hemocomponentes.

A entrevista da enfermeira (E02) evidencia que o problema da comunicação entre os membros das equipes multiprofissionais, é algo existente e exige dessas enfermeiras o desenvolvimento de estratégias para melhorar a comunicação com o intuito de minimizá-los ou saná-los.

[...] Olha dificuldade é em termos da equipe, eu acho, da equipe ser integrada, de todos falarem a mesma língua. Eu sinto que não é uma coisa única. Eu sinto isso. Acaba dificultando o trabalho. Não só das enfermeiras com os médicos como das enfermeiras com os técnicos. Acaba dificultando. Existe uma barreira. Discutir com a equipe o fato e as estratégias para corrigir e melhorar. Acho que essa é a melhor maneira. (E02)

Pode-se destacar que a comunicação eficaz é um elo que deve ser trabalhado pela enfermeira que atua gerenciando a equipe de enfermagem, visando sempre melhorar a assistência prestada aos que participam ativamente do processo de doação de sangue, como doadores, ou dos pacientes que necessitam dos hemocomponentes.

Com relação à busca pela melhoria na convivência entre os indivíduos que compõem as equipes e a implementação de ações que visam sanar a falta de comunicação e de companheirismo, Teixeira, Silva e Draganov (2018) verificou a necessidade dos mesmos aperfeiçoarem as suas competências no tocante a gestão de conflitos, desenvolvendo as suas habilidades em liderar e gerenciar diversas atividades, englobando o gerenciamento da equipe de enfermagem, a fim de prover uma qualidade no atendimento assistencial e na saúde organizacional, sendo imprescindível que o Enfermeiro tenha como habilidades: a capacidade de comunicação; observação; escuta; senso crítico; e a empatia para vislumbrar todas as faces de um conflito.

Conforme observado, os Enfermeiros que atuam no Serviço de Hemoterapia onde ocorreu o estudo são responsáveis por toda a gerência da equipe de enfermagem, pela gestão dos insumos e pela intermediação dos conflitos. Estes fatores estão alinhados com as atividades a serem desempenhadas pelos Enfermeiros atuando de forma gerencial e está aderente ao observado pelos pesquisadores Spagnol (2005), Lopes et al. (2018) e Ferracioli et al. (2020) em seus estudos, pois os técnicos de enfermagem que compõem as equipes buscam no enfermeiro o ponto de referência para sanar as dúvidas relacionadas com a assistência aos doadores e pacientes, o que faz com que o Enfermeiro exerça os aspectos pedagógicos, assistenciais e técnicocientíficos ao elucidar as dúvidas e treinar a sua equipe para corretamente assistir o paciente.

Pode-se verificar na fala da Enfermeira (E06), a preocupação em manter a equipe atualizada, focada, seguindo o que é preconizada pelas legislações vigentes.

[...] Nós temos os nossos procedimentos, os pops, que anualmente revisamos e fazemos uma leitura com todos os profissionais para garantir que todos os processos sejam executados conforme descrição daquela atividade. (E06) 
Ao lidar com a intermediação de conflitos, o enfermeiro está atuando de forma política e nas relações interpessoais, uma vez que a busca pela harmonia e coesão do grupo é requerida visando garantir um ambiente de trabalho agradável e uma união da equipe atuando no cuidado com o binômio doador- paciente.

No contexto, Lopes et al. (2018) e Ferracioli et al. (2020) destacam em seus estudos que o gerenciamento é uma ferramenta essencial no desenvolvimento do processo de trabalho, e é aplicável nas mais diversas áreas de atuação. Em relação ao gerenciamento em enfermagem, é utilizado como um norteador de ações, execuções, avaliações e planejamentos, sendo as competências gerenciais entendidas como um conjunto de conhecimentos, habilidades e atitudes que são implementados visando o aumento do desempenho.

Cabe destacar que a liderança, elemento da gerência, está intrinsecamente ligada nos serviços de saúde e é uma ferramenta amplamente empregada no dia a dia com as equipes, uma vez que a liderança está associada a uma comunicação efetiva, conhecimento, organização e a capacidade de estar à frente dos interesses da equipe, sendo por intermédio da liderança que o Enfermeiro assegura uma gerência assertiva.

Ribeiro, Santos e Meira (2006) verificou em sua pesquisa que, para o desenvolvimento das funções gerenciais do enfermeiro, é necessário que o mesmo fomente o trabalho em equipe, respeitando as individualidades, reconhecendo e estimulando as competências, capacidades e potencialidades de cada membro de sua equipe. É importante que, no desempenho cotidiano de suas atividades, o profissional de enfermagem integre a sua equipe nas soluções dos problemas, buscando ouvir as opiniões dos membros da equipe.

Outra atribuição gerencial do enfermeiro em Serviços de Hemoterapia de grande destaque está relacionada com a capacitação e com a atualização profissional da equipe de enfermagem do Serviço.

A participante E03 evidencia que as enfermeiras, onde ocorreu o presente estudo, realizam suas funções gerenciais relacionadas com treinamentos e capacitações, não apenas com a equipe pertencente ao Serviço de Hemoterapia, mas também com as demais equipes das diversas unidades de saúde do hospital universitário estudado, que necessitem de capacitação para o correto cumprimento das atividades relacionadas com o ato transfusional.

[...] Nós da agência fazemos um treinamento prático de forma rápida nas equipes. Hoje estou indo em todas as unidades. Faço orientações básicas de transfusão, entrego um formulário de transfusão e oriento as equipes de enfermagem. (E03)

Segundo Silva et al. (2015), o enfermeiro deve buscar a educação permanente da sua equipe por intermédio de treinamentos que abordem temáticas relacionadas com a prevenção de situações adversas, fomentando a participação em reuniões e eventos relacionados com temas e propostas de soluções de dilemas éticos relativos à Hemovigilância e notificação das reações adversas para que a equipe esteja sempre atualizada com as boas práticas realizadas empregadas amplamente nos demais Serviços de Hemoterapia e possam prestar a assistência aos pacientes em sua plenitude.

De acordo com Silveira, Bessa, Paes e Stipp (2017), outro ponto importante a ser desenvolvido nas funções gerenciais do enfermeiro é a capacidade de motivar a equipe de enfermagem, para isso há a necessidade de conhecer e entender profundamente cada um dos membros da equipe, suas particularidades e capacidades, visando à adoção de medidas motivacionais que refletirão positivamente na qualidade assistencial prestada e no aumento da produtividade.

\section{Considerações Finais}

Os profissionais que exercem suas funções nos Serviços de Hemoterapia buscam incansavelmente o atendimento das demandas dos pacientes acometidos por doenças das mais variadas, tendo o Enfermeiro como peça central na busca pelo 
correto funcionamento deste Serviço de forma harmônica e eficaz.

Logo, com a análise e discussão do contido nas entrevistas foi possível conhecer como as participantes do estudo desempenham suas funções gerenciais, com relação às ações do cotidiano do Serviço de Hemoterapia, podendo ser observado que as mesmas atuam: no gerenciamento da equipe de enfermagem; no gerenciamento de insumos e materiais de saúde específicos destes Serviços; na execução de treinamentos; no gerenciamento de conflitos; na atuação da melhoria da comunicação efetiva na equipe multiprofissional; e na assistência aos doadores e receptores dos hemocomponentes.

Com isso, infere-se, também, que às atividades gerenciais do Enfermeiro no cotidiano de um Serviço de Hemoterapia podem ser divididas em dois grupos, sendo o primeiro classificado como gerência positiva e que contempla a gestão de insumos, a gestão da equipe, a aquisição de materiais, a elaboração de termos de referência, a realização de treinamentos, e a busca pela atualização de assuntos afetos ao sangue e seus hemocomponentes, e o segundo classificado como gerência de tensão onde estão contidas a melhoria da comunicação, a interação entre os membros das equipes e a gestão de conflitos que possam vir a ocorrer internamente às equipes, fazendo com que estes profissionais se reinventem diariamente para que possam desenvolver as funções gerenciais em sua plenitude.

$\mathrm{O}$ desenvolvimento de pesquisas voltadas ao dimensionamento e a otimização de equipes de enfermagem atuando em Serviços de Hemoterapia são sugestões de trabalhos futuros a serem realizados, visando à busca por dados que subsidiem os enfermeiros nos pleitos pelo aumento de suas equipes para que possam não apenas prestar uma assistência de excelência aos mais necessitados, mas também que os mesmos possibilitem condições adequadas de trabalho para as suas equipes.

\section{Referências}

Almeida, R. G. S., Mazzo, A., Mendes, I. A. C., Trevizan, M. A., \& Godoy, S. (2011). Caracterização do atendimento de uma unidade de Hemoterapia. Revista Brasileira de Enfermagem, 64(6), 1082-6. https://doi.org/10.1590/S0034-71672011000600014.

Barbosa, S. M., Torres, C. A., Gubert, F. A., Pinheiro, P. N. C., \& Vieira, N. F. C. (2011). Enfermagem e a prática hemoterápica no Brasil: revisão integrativa. Acta Paul Enfermagem, 24(1), 132-6. https://doi.org/10.1590/S0103-21002011000100020.

Bardin, L. (2016). Análise de Conteúdo. Edições 70.

Beserra, M. P. P., Portela, M. P., Monteiro, M. P., Façanha, M. C., Adriano, L. S., \& Fonteles, M. M. F. (2014). Reações transfusionais em um hospital cearense acreditado: uma abordagem em hemovigilância. Arquivo de Medicina, 28(4), 99-103. http://scielo.pt/scielo.php?script=sci_arttext\&pid=S087134132014000400002 ?script=sci_arttext\&pid=S0871-34132014000400002.

Buozi, B. C., Lopes, C. T., Santos, E. R., Bergamasco, E. C., \& Murakami, B. M. (2019). Adequação das atividades da intervenção "administração de hemoderivados" da classificação das intervenções de enfermagem para pacientes adultos. Revista Mineira de Enfermagem, 23(4), e-1258. https://doi.org/10.5935/1415-2762.20190106.

Carneiro, V. S. M., Barp, M., \& Coelho, M. A. (2017). Hemoterapia e reações transfusionais imediatas: atuação e conhecimento de uma equipe de enfermagem. Revista Mineira de Enfermagem, 21, e-1031. https://doi.org/10.5935/1415-2762.20170041.

Faquetti, M. M., Rosa, R. L., Bellaguarda, M. L. R., Lazzari, D. D., Tholl, A. D., \& Moraes, C. L. K. (2014). Percepção dos receptores sanguíneos quanto ao processo transfusional. Revista Brasileira de Enfermagem, 67(6), 936-41. http://dx.doi.org/10.1590/0034-7167.2014670611.

Ferracioli, G. V., Oliveira, R. R., Souza, V. S., Teston, E. F., Varela, P. L. R., \& Costa, M. A. R. (2020). Competências gerenciais na perspectiva de enfermeiros do contexto hospitalar. Enfermagem em Foco, 11(1), 15-20. https://doi.org/10.21675/2357-707X.2020.v11.n1.2254.

Frantz, S. R. S., Vargas, M. A. O., Pires, D. E. P., Brito, M. J. M., Bitencourt, J. V. O. V., \& Ribeiro, G. (2020). Nursing work and competence in hemotherapy services: an ergological approach. Revista Brasileira de Enfermagem, 73(3), e20180775. https://doi.org/10.1590/0034-7167-2018-0775.

Frantz, S. R. S., \& Vargas, M. A. O. (2021). Renormalização do trabalho do enfermeiro em hemoterapia: entre o prescrito e o real. Texto \& Contexto Enfermagem, 30, e20190060. https://doi.org/10.1590/1980-265X-TCE-2019-0060.

Greco, R. M. (2004). Ensinando a administração em enfermagem através da educação em saúde. Revista Brasileira de Enfermagem, 57(4), 504-7. https://doi.org/10.1590/S0034-71672004000400026.

Lopes, L. F., Pontelli, B. P. B., \& Oliveira, R. E. M. (2018). Gerência de enfermagem e trabalho em equipe na atenção básica: uma revisão de literatura. Revista Fafibe Online, 11(1), 108-16. https://www.unifafibe.com.br/revista/index.php/fafibeonline/article/view/563.

Ribeiro, M., Santos, S. L., \& Meira, T. G. B. M. (2006). Refletindo sobre liderança em enfermagem. Escola Ana Nery Revista de Enfermagem, 10(1), 109-15. https://doi.org/10.1590/S1414-81452006000100014. 
Research, Society and Development, v. 11, n. 2, e35211225628, 2022

(CC BY 4.0) | ISSN 2525-3409 | DOI: http://dx.doi.org/10.33448/rsd-v11i2.25628

Rohr, J. I., Boff, D., \& Lunkes, D. S. (2012). Perfil dos candidatos inaptos para doação de sangue no serviço de hemoterapia do Hospital Santo Ângelo, RS, Brasil. Revista de Patologia Tropical, 41(1), 27-35. https://doi.org/10.5216/rpt.v41i1.17750.

Sanches, V. F., Christovam, B. P., \& Silvino, Z. R. (2006). Processo de trabalho do gerente de enfermagem em unidade hospitalar - uma visão dos enfermeiros. Escola Ana Nery Revista de Enfermagem, 10(2), 214-20. https://doi.org/10.1590/S1414-8145200600020000.

Santos, N. L. P., Stipp, M. A. C., Silva, A. L. A., Moreira, M. C., \& Leite, J. L. (2013). O cuidado de enfermagem aos doadores de sangue - a perspectiva da integralidade. Escola Ana Nery Revista de Enfermagem (impresso), 17(4), 661-7. https://doi.org/10.5935/1414-8145.20130009.

Silva, F. C. G., Senne, E. C. V., Peixoto, P. B., Paiva, L., Olivo, R. A., \& Szymaniak, N. P. (2015). Análise dos incidentes de transfusão sanguínea em pacientes hospitalizados. Liph Science, 2(1), 41-55. https://www.researchgate.net/publication/289397947.

Silveira, C. D., Bessa, A. T. T., Paes, G. O., \& Stipp, M. A. C. (2017). Gestión del equipo de enfermería: factores asociados a la satisfacción en el trabajo. Enfermería Global, 16(3), 208-23. https://doi.org/10.6018/eglobal.16.3.249471.

Spagnol, C. A. (2005). (Re)pensando a gerência em enfermagem a partir de conceitos utilizados no campo da Saúde Coletiva. Ciência \& Saúde Coletiva, 10(1), 119-27. https://doi.org/10.1590/S1413-81232005000100019.

Teixeira, N. L., Silva, M. M., \& Draganov, P. B. (2018). Desafios do enfermeiro no gerenciamento de conflitos dentro da equipe de enfermagem. Revista de Administração em Saúde, 18(73). http://dx.doi.org/10.23973/ras.73.138.

Torres, R. C., Xavier, A. F. S., Sousa, P. H. S. F., Silva, M. M. L., Andrade, A. F. S. M., Santos Junior, P. C. C., Costa, M. F., \& Azevedo, M. V. C. (2021). Atuação do enfermeiro em hemoterapia: a visão do formado. Brazilian Journal of Development, 7(2), 16000-14. https://doi.org/10.34117/bjdv7n2-294.

Ventura, P. F. E. V., Freire, E. M. R., \& Alves, M. (2016). Participação do enfermeiro na gestão de recursos hospitalares. Revista Eletrônica de Gestão \& Saúde, 7(1), 126-47. https://periodicos.unb.br/index.php/rgs/article/view/3398. 